\title{
Interventions nutritionnelles chez la femme allaitante infectée par le VIH
}

\author{
Saurabh Mehta ${ }^{a, b}$ Julia L. Finkelstein ${ }^{a}$ Wafaie W. Fawzi ${ }^{a, b}$ \\ Départements de a Nutrition et ${ }^{b}$ Épidémiologie, École de Santé Publique de Harvard, Boston, Mass., États-Unis
}

\section{Mots-clés}

Nutrition $\cdot \mathrm{VIH} \cdot$ Sida $\cdot$ Supplémentation multivitaminique $\cdot$ Allaitement maternel

\section{Résumé}

L'allaitement maternel est la référence internationale en matière de nutrition du nourrisson, car il est connu qu'il réduit les risques de morbidité et de mortalité infantiles et ameliore la croissance et le développement de l'enfant. Pourtant, dans le contexte de l'infection à $\mathrm{VIH}$, l'allaitement maternel augmente significativement le risque de transmission mère-enfant (TME) du virus, de façon proportionnelle à la durée de l'allaitement. En conséquence, I'Organisation Mondiale de la Santé recommande que les mères infectées par le VIH n'allaitent pas quand une alimentation de remplacement est acceptable, faisable, financièrement abordable, durable et sûre. Malheureusement, l'adoption de l'alimentation par formule n'est pas largement répandue dans des régions en développement telles que l'Afrique sub-saharienne, partiellement en raison de son coût élevé et également de la stigmatisation sociale que suscite le fait de ne pas allaiter son enfant. De plus, dans des contextes où l'accès à des conditions hygiéniques adéquates est limité, l'alimentation par formule peut aboutir à une augmentation de la morbidité et de la mortalité infantile. En conséquence, l'allaitement maternel continue à être à l'origine d'une proportion substantielle des infections pédiatriques à VIH dans les régions en développement au plan mondial. Certaines études observationnelles ayant examiné le rôle du statut nutritionnel dans la transmission verticale du VIH ont montré qu'une basse concentration sérique de vitamine $A$ était associée à un accroissement du risque de TME. Lors de deux essais menés au Malawi et en Afrique du Sud, la vitamine A n'a cependant exercé aucun effet significatif sur la TME du VIH, tandis que des essais réalisés en Tanzanie et au Zimbabwe ont montré qu'une supplémentation maternelle en vitamine $A$ accroissait significativement ce risque. En Tanzanie, une supplémentation multivitaminique (vitamines $B, C$ et $E$ ) maternelle a réduit le risque de transmission du VIH par l'allaitement chez des femmes compromises au plan nutritionnel et immunologique et a exercé des bénéfices additionnels pour la santé tels qu'une diminution de l'incidence des fausses couches et une réduction du taux de progression de la maladie chez les mères. Globalement, il n'existe aucune donnée en faveur d'une supplémentation en vitamine A chez les femmes enceintes infectées par le VIH. Une supplémentation multivitaminique incluant les vitamines $B, C$ et $E$ est cependant fortement recommandée. De nouvelles recherches sont justifiées afin de déterminer le rôle d'autres nutriments dans la transmission verticale du VIH par l'intermédiaire de l'allaitement maternel et dans la santé générale des femmes allaitantes infectées par le VIH et de leurs nourrissons. Dans les contextes où des alternatives à l'allaitement maternel ne sont pas envisageables, un allaitement maternel exclusif est recommandé pendant 6 mois pour les enfants nés d'une mère infectée par le $\mathrm{VIH}$, ainsi qu'un sevrage rapide et des aliments de remplacement optimaux pour la santé du nourrisson.

Copyright @ 2007 Nestec Ltd., Vevey/S. Karger AG, Basel

\section{KARGER}

Fax +4161306 1234 E-Mail karger@karger.ch www.karger.com (c) 2007 Nestec Ltd., Vevey/S. Karger AG, Basel 0250-9644/07/0651-0039\$23.50/0

Accessible en ligne à: www.karger.com/anf
Wafaie W. Fawzi, MD, DrPH

Department of Nutrition, Building II Room 329A

Harvard School of Public Health, 651 Huntington Avenue

Boston, MA 02115 (USA)

Tel. +1 617432 5299, Fax +1 617432 2435, E-Mail mina@hsph.harvard.edu 


\section{Introduction}

Des estimations indiquent quau plan mondial, 1800 enfants sont infectés par le VIH chaque jour, avec environ 650000 nouvelles infections pédiatriques et 500000 décès liés au sida chaque année [1,2]. L'Afrique sub-saharienne subit la majorité de la charge pathologique pédiatrique globale liée au VIH/sida, représentant plus de $85 \%$ des nouvelles infections à VIH d'enfants au plan mondial. En 2003, le sida a été responsable de $6,5 \%$ des décès d'enfants de moins de 5 ans en Afrique, en augmentation par rapport à un taux de $2 \%$ en 1990 [2]. La majorité des infections pédiatriques à VIH sont contractées par transmission mère-enfant (TME) in utero, pendant l'accouchement ou au cours de l'allaitement.

Dans les pays industrialisés, des stratégies telles qu'un traitement antirétroviral et une alimentation exclusive par formule ont réduit avec succès le taux de transmission verticale du VIH à moins de 2\% [3]. Cependant, en l'absence de traitement antirétroviral et d'intervention nutritionnelle dans les pays en développement, les enfants sont exposés à un risque de 25 à $48 \%$ de contracter l'infection par l'intermédiaire de leur mère [4]. De fait, l'allaitement maternel continue à être responsable de 300000 nouvelles infections pédiatriques annuellement dans les pays en développement, soit environ $40 \%$ de l'ensemble des TME du VIH [5].

En conséquence, l'Organisation Mondiale de la Santé recommande que les mères infectées par le VIH n'allaitent pas leur enfant «quand une alimentation de remplacement est acceptable, faisable, financièrement abordable, durable et sûre» [6]. Une alimentation exclusive de remplacement n'est cependant pas généralement envisageable dans des pays moins développés en raison de l'insuffisance des ressources en eau potable, des conditions hygiéniques inadéquates, du coût élevé des formules, de la stigmatisation sociale et de l'augmentation du risque de morbidité et de mortalité infantiles [7-9]. De plus, les régions en développement sont confrontées à la rareté des services de soins prénataux, et en particulier des conseils et tests volontaires concernant le VIH, et de l'accès au traitement antirétroviral. Par exemple, et bien que les estimations de la séroprévalence du VIH aillent de 15 à 30\% dans les centres de soins prénataux de nombreux pays africains subsahariens, la couverture par des services de prévention de la TME est estimée à moins de $6 \%$ en moyenne [2]. Dans ce contexte, des interventions sanitaires préventives, incluant des conseils pour l'alimentation et la nutrition des nourrissons, et la fourniture d'un traitement antirétroviral deviennent de plus en plus importantes afin de réduire la TME du VIH et de stimuler des améliorations de la santé maternelle et infantile.

Dans le présent article, nous examinerons le rôle de la nutrition chez les femmes allaitantes infectées par le VIH et leurs enfants. Nous débuterons par un bref résumé des bénéfices de l'allaitement maternel et des besoins nutritionnels globaux au cours de l'allaitement. La relation entre nutrition, VIH et allaitement maternel sera explorée de façon détaillée par l'analyse des données disponibles issues d'études observationnelles et d'essais randomisés. Enfin, nous conclurons par une discussion des implications de diverses observations pour les pratiques cliniques et de santé publique.

\section{Allaitement par les mères non infectées par le VIH}

\section{Bénéfices de l'allaitement maternel}

Il est bien établi que l'allaitement maternel est la norme internationale de nutrition du nourrisson en raison de ses bénéfices tant pour la mère que pour l'enfant. Les bénéfices pour la mère comportant l'échelonnement des enfants dans le temps, le confort psychologique, un retour plus rapide au poids corporel antérieur à la grossesse, une réduction du risque d'hémorragie post-partum et une diminution de l'incidence des cancers du sein et de l'ovaire avant la ménopause [10].

L'allaitement maternel confère des bénéfices nutritionnels et immunologiques au nourrisson, en particulier une diminution de la morbidité et de la mortalité dues à des affections diarrhéiques et des infections respiratoires aiguës [11]. Par exemple, dans les pays en développement, la mortalité par affections diarrhéiques et pneumopathies infectieuses est trois à dix fois plus élevée chez les nourrissons alimentés par formule que chez ceux exclusivement allaités par leur mère $[12,13]$. De fait, selon une récente analyse, les seuls programmes de promotion de l'allaitement maternel pourraient directement prévenir annuellement un nombre estimé de 1,3 million de décès d'enfants au plan mondial [14]. Cette augmentation du risque de morbidité et de mortalité d'origine infectieuse associée à l'alimentation par formule s'est avérée persister même dans le contexte d'un accès modeste à de l'eau potable et à des installations sanitaires [15]. Une explication de cette observation pourrait être que l'alimentation par formule pourrait priver l'enfant des bénéfices immunologiques protecteurs de l'allaitement maternel, dont l'apport de cytokines et d'anticorps [1618]. L'allaitement maternel est également bénéfique pour la croissance et le développement du nourrisson, en particulier celui dont une amélioration des fonctions neurocognitives des prématurés de très petit poids de naissance [19], probablement imputable en la teneur en acides gras polyinsaturés du lait maternel [20]. De plus, l'allaitement maternel réduit le risque d'otite moyenne et d'infection urinaire chez le nourrisson [10, $13,21]$.

\section{Besoins nutritionnels au cours de l'allaitement maternel}

Afin de pouvoir soutenir le métabolisme maternel ainsi que la croissance et le développement du nourrisson, les besoins nutritionnels de la mère sont plus élevés au cours de l'allaitement que lors de la période prénatale. Par exemple, le nourrisson double son poids de naissance au cours des 4 à 6 premiers mois de sa vie [22]. Les besoins maternels en macro- 
nutriments sont également plus élevés au cours de l'allaitement et sont directement proportionnels à l'intensité et à la durée de celui-ci. Les recommandations actuelles préconisent un apport additionnel de $500 \mathrm{kcal} /$ jour au cours des 6 premiers mois de l'allaitement et de $400 \mathrm{kcal} /$ jour par la suite $[22,23]$.

Les besoins en micronutriments de la mère et du nourrisson sont également accrus au cours de la période d'allaitement. Dans le contexte d'une consommation énergétique inadéquate, les apports maternels en calcium, magnésium, zinc, folate et vitamine $B_{6}, B_{12}$ et $D$ peuvent être réduits [24]. Une supplémentation maternelle en vitamine $B_{12}$ et $D$ peut être notamment nécessaire au cours de la période d'allaitement, particulièrement quand le régime maternel exclut les produits d'origine animale $[22,24]$. Les recherches menées à ce jour ont été principalement centrées sur le rôle de la vitamine $\mathrm{B}_{12}$ et des folates, en raison de leur association à des affections congénitales manifestes par carence, mais les effets de carences en micronutriments sur la santé de la mère et du nourrisson au cours de l'allaitement ont été très insuffisamment examinés.

\section{Allaitement par les mères infectées par le VIH}

\section{TME du VIH par l'intermédiaire du lait maternel}

L'hypothèse de la transmission verticale du VIH par le lait maternel a été initialement émise à la suite de l'observation d'infections à VIH chez des nourrissons allaités par des mères ayant présenté une séroconversion postnatale à la suite d'une exposition sexuelle ou d'une transfusion sanguine [25, 26]. Cette hypothèse a été confortée par des descriptions consécutives de transmission du VIH par des nourrices et du lait de femme issus de regroupement de dons chez des nourrissons exempts de toute autre source connue d'exposition au VIH [27].

Comme mentionné plus haut, des interventions telles qu'un traitement antirétroviral et une alimentation exclusive par formule ont réduit l'incidence de la TME du VIH à moins de $2 \%$ dans des pays industrialisés tels que les États-Unis [3], mais, dans les pays en développement, où l'allaitement maternel et le VIH sont largement prévalents, la contribution proportionnelle de la TME au poids de l'infection à VIH est considérablement plus importante.

Des facteurs liés à la mère et au nourrisson influencent le risque de TME du VIH par l'intermédiaire de l'allaitement. Par exemple, des caractéristiques maternelles telles qu'un taux élevé d'ARN du VIH dans le plasma et le lait, une immunodéficience (par exemple faible compte de lymphocytes CD4), une forme clinique avancée de la maladie et un mauvais état nutritionnel augmentent la probabilité de transmission verticale du VIH [28-30]. Des caractéristiques virales peuvent également influencer le risque de TME du virus. Par exemple, une étude menée chez des paires se composant de mères infectées par le
VIH et de leurs nourrissons a montré que seuls les virus qui n'induisaient pas un syncytium et présentaient un tropisme plus élevé pour les macrophages ont été transmis aux enfants allaités [31, 32]. La présence d'une affection inflammatoire telle qu'une mastite maternelle et un abcès du sein contribuent également au risque d'exposition du nourrisson au VIH et de transmission consécutive de ce virus [33,34]. Des facteurs liés au nourrisson tels qu'une candidose et des ulcères buccaux [33, 34] et l'intégrité épithéliale des voies digestives [35] influencent également le risque d'exposition au VIH et de transmission du virus [33, 34].

Les modalités de l'alimentation du nourrisson modulent également le risque de TME du VIH. Plusieurs études ont démontré que le taux de transmission du VIH augmentait de façon cumulative avec la durée de l'allaitement maternel pendant toute la durée de la période post-natale [36, 37]. Cette observation est probablement imputable à l'accroissement de l'exposition du nourrisson au virus. Par exemple, dans l'étude multicentrique Breastfeeding and HIV International Transmission Study, le taux de transmission du VIH par l'allaitement maternel à des nourrissons séronégatifs pour ce virus à l'âge de 4 semaines a été de $0,74 \%$ par mois, soit un risque de $4 \%$ par 6 mois d'allaitement maternel [5].

Le moment de la séroconversion maternelle peut également influencer le risque de transmission verticale du VIH. Par exemple, lors d'une étude prospective de cohorte menée au Rwanda, les investigateurs ont observé un taux de transmission de $80 \%$ du VIH chez les nourrissons nés de mères qui avaient présenté une séroconversion dans les 3 mois suivant l'accouchement, contre $40 \%$ chez ceux dont les mères avaient été infectées de 4 à 21 mois post-partum [38]. La transmission du VIH par l'intermédiaire de l'allaitement a été cependant rapportée après de longs délais tels que 36 mois post-partum [39].

Le mode de nutrition de l'enfant peut également influencer la probabilité de TME du VIH; par exemple, plusieurs études ont montré que le risque de transmission du VIH avait été le plus élevé avec l'allaitement mixte comparativement à un allaitement exclusif à court terme et une nutrition par formule [4042]. Ces études, ainsi que d'autres ayant porté sur des facteurs maternels et infantiles liés au risque de transmission verticale du VIH par l'allaitement, ont été analysées de façon détaillée par Downs et Cooper [43].

\section{Effets de l'allaitement maternel sur le statut nutritionnel et la mortalité des femmes infectées par le VIH}

Plusieurs études observationnelles et essais randomisés ont été menés afin de déterminer les effets de l'allaitement sur la santé des femmes infectées par le VIH. Par exemple, une étude observationnelle menée en Afrique du Sud a comparé le statut nutritionnel et la composition corporelle entre 44 femmes allaitantes négatives pour le VIH et 17 positives pour ce virus. Aucune différence significative n'a été observée entre les deux 
groupes quant aux mesures du poids corporel, de l'indice de masse corporelle, de la circonférence au milieu du bras ou de l'épaisseur du pli cutané au triceps [44].

Lors d'un essai randomisé mené au Kenya, 425 femmes infectées par le VIH ont été aléatoirement affectées à un groupe allaitement maternel ou à un groupe alimentation par formule. Les investigateurs ont comparé le poids des mères initialement (2 semaines à 3 mois post-partum) et au cours du suivi (5-9 mois post-partum) et ont déterminé la mortalité tout au long de la période de suivi. Les mères allaitantes ont perdu en moyenne $0,17 \mathrm{~kg} / \mathrm{mois}$ tandis que celles du groupe alimentation par formule n'ont pas perdu de poids en moyenne $(\mathrm{p}=$ $0,03)$ [45]. Le risque de décès a également été trois fois plus élevé chez les femmes allaitantes que chez celles du groupe alimentation par formule. Les investigateurs ont supposé que l'accroissement observé de la mortalité dans le groupe allaitement maternel était probablement dû à une déplétion en nutriments. Une faible observance a pu cependant atténuer l'association observée entre le mode d'alimentation du nourrisson et le risque consécutif de décès maternel, ce qui limite les possibilités d'interprétation des observations. Par exemple, $4 \%$ des femmes affectées au groupe allaitement maternel n'ont jamais allaité leurs nourrissons et $29 \%$ de celles affectées au groupe nutrition par formule ont également allaité leurs nourrissons. Des différences significatives des caractéristiques initiales ont été également constatées entre les deux groupes, en particulier une charge plasmatique virale médiane plus élevée chez les femmes affectées au groupe allaitement maternel, ce qui a pu augmenter le risque de TME du VIH dans ce groupe. De plus, le taux de transmission intra-utérine du VIH a été plus élevé dans le groupe allaitement maternel, ce qui a pu être imputable à une forme avancée de la maladie chez les femmes allaitantes. Les auteurs n'ont toutefois fourni aucune information sur la progression clinique de la maladie dans les deux groupes [46]. De plus, peu de mécanismes biologiques plausibles pourraient expliquer un effet de l'allaitement sur la mortalité maternelle [47].

L'association entre allaitement maternel et risque de décès n’a pas été retrouvée lors d'autres études sur le terrain menées chez des femmes allaitantes positives pour le VIH. Par exemple, une étude menée chez 566 femmes infectées par le VIH en Afrique du Sud a indiqué que les modalités d'alimentation du nourrisson n'avaient pas influencé le risque de décès maternel: 2 des $410(0,49 \%)$ femmes ayant allaité sont décédées comparativement à 3 des $156(1,92 \%)$ qui n’avaient jamais allaité [47]. De même, dans une cohorte de femmes en Tanzanie, le statut vis-à-vis de l'allaitement maternel ou la durée de celui-ci n'ont pas été liés au risque de décès, de perte de poids, d’anémie ou de modifications du compte des lymphocytes CD4 chez les mères [48]. De plus, aucune association n'a été observée entre la présence ou l'absence d'allaitement maternel et le risque de décès des mères lors d'études menées en Zambie [49] et au Malawi [50], ou lors d'une étude multicentrique menée par le Breastfeeding and HIV International Transmission Study Group [51].
Rôle de la nutrition chez les femmes allaitantes infectées par le $\mathrm{VIH}$

Les bénéfices de l'amélioration de la nutrition maternelle devraient être doubles. Tout d'abord, elle peut réduire la TME du VIH par l'intermédiaire de l'allaitement, à titre d'intervention d'appoint chez les femmes recevant déjà un traitement antirétroviral ou de seule stratégie chez celles qui se situent à un stade de la maladie où elles ne sont pas éligibles pour un traitement antirétroviral. Deuxièmement, les interventions nutritionnelles amélioreront probablement la santé et la qualité de vie de la mère ainsi que la croissance et le développement du nourrisson. Des essais contrôlés menés chez des hommes et des femmes infectés par le VIH au Canada [52], en Thaïlande [53] et en Tanzanie [54] ainsi que plusieurs études observationnelles bien conçues [55] ont par exemple démontré l'effet bénéfique d'une supplémentation en micronutriments sur la progression clinique de l'infection à VIH. Des interventions nutritionnellestelles qu'une supplémentation en micronutriments peuvent donc être un appoint utile au traitement antirétroviral ou constituer la seule stratégie chez des femmes allaitantes infectées par le VIH afin de susciter des améliorations de la santé maternelle et infantile.

Il existe plusieurs mécanismes potentiels par lesquels une supplémentation maternelle en nutriments peut être bénéfique tant pour la mère que pour le nourrisson, comme une amélioration de l'état de santé et une réduction du risque de transmission verticale du VIH. Les micronutriments sont par exemple importants pour l'intégrité épithéliale des tissus mammaires maternels, qui influence l'exposition du nourrisson au virus et le risque de transmission de celui-ci. Plusieurs études ont notamment démontré qu'une carence en vitamine A dans l'épithélium des cellules mammaires etait responsable de colonies cellulaires de forme irrégulière avec une anomalie de la polarité ou des protéines de jonction intercellulaire [5658]. De même, une supplémentation en micronutriments peut renforcer l'intégrité des muqueuses des voies digestives du nourrisson et influencer les fonctions des lymphocytes $\mathrm{T}$ et $\mathrm{B}$ de la mère et du nourrisson [59-61]. Ces effets peuvent réduire par la suite la sensibilité à l'infection à VIH. Une étude menée en Afrique du Sud chez des enfants nés de mères infectées par le VIH a montré par exemple que des réponses cellulaires à lymphocytes $\mathrm{T}$ auxiliaires aux protéines d'enveloppe du VIH dans le sang du cordon ombilical avait été associée à une protection contre la transmission de ce virus pendant l'accouchement et l'allaitement [62]. Le lait maternel confère également des bénéfices nutritionnels et immunologiques au nourrisson allaité, dont des facteurs pouvant le protéger contre l'infection à VIH, tels qu'immunoglobulines, lactoferrine, lysozyme, oligosaccharides et glycosaminoglycanes. Les bénéfices de ces facteurs immunologiques présents dans le lait maternel peuvent être encore accrus par des améliorations du statut nutritionnel maternel par une supplémentation en micronutriments $[59,63]$. 
Effet du statut nutritionnel maternel sur la TME du VIH par

l'intermédiaire de l'allaitement

\section{Études observationnelles}

Peu d'études ont exploré le rôle de la nutrition comme déterminant de paramètres de l'état de santé chez des femmes allaitantes positives pour le VIH et leurs enfants. Des études observationnelles ont principalement examiné le rôle de micronutriments dans la TME globale du VIH, avec un centrage sur la vitamine A. Pour les objectifs du présent article, nous avons analysé des études observationnelles ayant déterminé la TME globale du VIH, ce qui inclut les contaminations in utero, pendant l'accouchement et lors de l'allaitement. Ces études n'étaient pas spécifiquement destinées à examiner la transmission du VIH par l'intermédiaire de l'allaitement, mais des paramètres sélectionnés ont été évalués à la $6^{\text {ème }}$ semaine suivant l'accouchement et pouvaient inclure certaines contributions de cette voie de TME.

Une étude observationnelle a été menée au Kenya afin d'évaluer la relation entre le statut maternel en vitamine A et le risque de transmission verticale du VIH. Les investigateurs ont analysé 212 échantillons de lait provenant de 107 femmes allaitantes infectées par le VIH. Dans la population globale, ils n'ont observé aucune association entre le statut maternel en vitamine A et la présence d'ADN du VIH dans le lait. Une concentration sérique faible en vitamine $\mathrm{A}(<20 \mu \mathrm{g} / \mathrm{dl})$ a été toutefois associée à une multiplication par 20 du risque de présence détectable d'ADN du VIH dans le lait dans un sousgroupe de femmes immunodéficientes (compte des lymphocytes $\mathrm{CD} 4<400 / \mathrm{mm}^{3}$; odds ratio $=19,7$, intervalle de confiance à $95 \%$ [IC 95\%] 2,1, 188,5) [64].

Une étude a été menée au Malawi afin d'examiner la relation entre le statut maternel en vitamine A et la transmission verticale du VIH. Le taux cumulé de TME du virus a été de $21,9 \% 12$ mois après l'accouchement dans une population de 338 femmes enceintes infectées par le VIH. Les taux observés de transmission verticale du VIH ont été les plus élevés chez les femmes qui présentaient les concentrations sériques les plus basses de vitamine A [65].

L'association observée entre le statut maternel en vitamine A et le risque de transmission verticale du VIH pourrait être imputable à d'autres biais. Une infection peut contribuer, par exemple, à une diminution de la mobilisation des stocks hépatiques de vitamine $\mathrm{A}$ et donc à une réduction de la concentration sérique de celle-ci $[66,67]$. Une faible concentration de vitamine A peut donc être un marqueur d'un stade avancé de l'infection à VIH et expliquer l'accroissement observé du risque de transmission du virus. Il peut également exister des situations résiduelles à l'origine de biais dus à d'autres facteurs non contrôlés lors de ces études observationnelles, dont d'autres carences nutritionnelles, des infections opportunistes et un accès limité aux soins. Une autre limitation de ces études observationnelles est l'absence d'examen de la voie ou du moment de la transmission verticale du VIH, ce qui limite l'interprétation des observations concernant la contri- bution spécifique de l'allaitement maternel à la TME du virus.

\section{Études de supplémentation}

Les observations et limitations des études susmentionnées ont motivé la conduite d'essais contrôlés randomisés afin de mieux explorer la relation entre une supplémentation nutritionnelle et des paramètres de la santé chez des femmes positives pour le VIH et leurs enfants.

Lors d'un essai mené en Afrique du Sud, 728 femmes enceintes infectées par le VIH ont été aléatoirement affectées à une supplémentation quotidienne par vitamine $\mathrm{A}$ ou à un placebo pendant toute la durée de la période prénatale. La supplémentation en vitamine A se composait de la prise quotidienne de 5000 UI de vitamine A (1,67 mg d'équivalents rétinol) et de $30 \mathrm{mg}$ de $\beta$-carotène (5 $\mathrm{mg}$ d'équivalents rétinol) pendant la totalité du troisième trimestre de la grossesse, et de l'administration de 200000 UI de vitamine A à l'accouchement. Le risque d'infection à VIH et le taux de mortalité foetale ou infantile n'ont pas différé entre les deux groupes au cours des 3 mois suivant l'accouchement [68]. Une supplémentation prénatale en vitamine A n'a pas réduit le risque de transmission du VIH par l'allaitement maternel dans une sous-population de 551 femmes enceintes infectées par le VIH qui avaient été suivies jusqu'au $15^{\text {ème }}$ mois post-partum ou jusqu'à l'arrêt de l'allaitement [42]. Une analyse du taux d'ARN du VIH dans des échantillons de lait provenant d'un sousgroupe de 79 femmes infectées par le VIH a montré que la charge virale avait été étroitement associée au risque de TME du virus. Une supplémentation maternelle en vitamine A n'a cependant pas significativement réduit le risque de transmission verticale du VIH [69].

Lors d'un essai mené au Malawi, 697 femmes enceintes infectées par le VIH ont été aléatoirement affectées à l'administration quotidienne de fer et de folate tout au long de la grossesse jusqu'à l'accouchement, seuls ou en association à la vitamine $\mathrm{A}$ ( $3 \mathrm{mg}$ de rétinol). Toutes les femmes ont également reçu $30 \mathrm{mg}$ de rétinol 6 semaines après l'accouchement dans le cadre des soins standards. Aucune différence n'a été constatée entre les deux groupes quant au taux global de TME du VIH à 6 semaines (vitamine A 26,6\%, placebo $27,8 \% ; \mathrm{p}=0,76$ ) ou à 24 mois post-partum (vitamine A 27,7\%, placebo $32,8 \%$; $p=0,21$ ). Une supplémentation maternelle en vitamine A a cependant été associée à une diminution du taux de TME du VIH dans un sous-groupe de nourrissons qui étaient négatifs pour le VIH à 6 semaines selon la réaction en chaîne à la polymérase (PCR). Cet effet observé a paru attribuable à une réduction de la transmission du VIH par l'allaitement $(2,8$ contre $7,7 \%$ à 24 mois; $\mathrm{p}=0,04)$. Cependant, le statut vis-à-vis de l'allaitement maternel n’a pas été spécifiquement examiné lors de cet essai, ce qui limite l'interprétation des résultats [70]. De plus, les auteurs ont observé qu'une supplémentation maternelle en vitamine A au cours de la grossesse n'avait pas significativement influencé le degré de charge du lait maternel en VIH dans un sous-groupe de ces femmes [33]. 
Au cours de l'essai ZVITAMBO mené au Zimbabwe, des femmes infectées par le VIH et leurs nourrissons ont été aléatoirement affectés selon un plan bifactoriel à l'administration quotidienne d'une dose unique élevée de vitamine $\mathrm{A}$ et/ou à un placebo dans les 96 heures suivant l'accouchement. Les quatre groupes d'intervention étaient les suivants: vitamine A chez la mère et le nourrisson, vitamine $\mathrm{A}$ chez la mère et placebo chez le nourrisson, placebo chez la mère et vitamine A chez le nourrisson et placebo chez la mère et le nourrisson. Au total, 2060 nourrissons négatifs pour le VIH ont été suivis pendant toute la durée de la période post-partum afin d'évaluer le taux de transmission post-natale de ce virus (séroconversion chez les nourrissons négatifs pour le VIH à 6 semaines post-partum). Au cours de la période de suivi, une séroconversion est survenue chez $12,1 \%$ des nourrissons; une supplémentation en vitamine A à dose unique élevée n'a pas réduit le risque de transmission post-natale du VIH [71]. La vitamine A n'a également exercé aucun effet sur la mortalité chez les nourrissons initialement positifs pour le VIH selon la PCR. Une supplémentation néonatale en vitamine A a réduit de $28 \%$ la mortalité $(\mathrm{p}=0,01)$ chez ceux initialement négatifs selon la PCR mais positifs selon ce même examen à 6 semaines, bien que la supplémentation maternelle n'ait exercé aucun effet sur la mortalité chez ces nourrissons. En revanche, la supplémentation maternelle et infantile en vitamine A a résulté en un doublement significatif de la mortalité ( $\mathrm{p} \leq$ $0,05)$ chez les nourrissons qui étaient séronégatifs à 6 semaines.

Dans l'étude TOV (Trial of Vitamins) menée à Daar es Salam, Tanzanie, 1078 femmes enceintes infectées par le VIH ont été aléatoirement affectées à 12-27 semaines de grossesse selon un plan bifactoriel à l'administration quotidienne de vitamine $\mathrm{A}$, de multivitamines, de vitamine A et de multivitamines ou d'un placebo. Le supplément en vitamine A se composait de $30 \mathrm{mg}$ de $\beta$-carotène $(5 \mathrm{mg}$ d'équivalents rétinol) et de 5000 UI de vitamine A préformée (1,667 mg d'équivalents rétinol); le supplément multivitaminique se composait d'un complexe de vitamines B ainsi que des vitamines $\mathrm{C}$ et E (20 mg de thiamine, $20 \mathrm{mg}$ de riboflavine, $25 \mathrm{mg}$ de vitamine $\mathrm{B}_{6}, 100 \mathrm{mg}$ de niacine, $50 \mu \mathrm{g}$ de vitamine $\mathrm{B}_{12}, 500$ $\mathrm{mg}$ de vitamine $\mathrm{C}, 30 \mathrm{mg}$ de vitamine $\mathrm{E}$ et $0,8 \mathrm{mg}$ d'acide folique). Les femmes du groupe supplémenté en vitamine A ont également reçu une dose orale de vitamine A (200 000 UI) à l'accouchement, tandis que celles des groupes multivitamines et placebo ont reçu un supplément placebo. Dans le cadre des soins standards, toutes les femmes ont également reçu $120 \mathrm{mg}$ de fer ferreux et $5 \mathrm{mg}$ de folates quotidiennement sous forme de comprimés et $300 \mathrm{mg}$ de chloroquine par semaine à titre de prévention du paludisme. Les suppléments multivitaminiques (risque relatif $[\mathrm{RR}]=0,95$, IC 95\% 0,73, 1,24) et en vitamine A $(\mathrm{RR}=1,06$, IC 95\% 0,81, 1,39) n'ont pas influencé le risque de transmission du VIH ou la survie des nourrissons jusqu'à 6 semaines post-partum. La supplémentation maternelle en multivitamines, mais non en vitamine A, a augmenté le poids de naissance des nourrissons [72] et a résulté en une réduction significative d'environ $40 \%$ des risques de fausses cauches, de petit poids de naissance $(<2500 \mathrm{~g})$, de prématurité sévère ( $<34$ semaines de gestation) et de retard de croissance intra-utérine [73]. Les multivitamines, mais non la vitamine $\mathrm{A}$, ont également entrainé une augmentation significative des comptes maternels de lymphocytes CD4, CD8 et CD3 [73].

Lors de l'essai TOV, une supplémentation maternelle en vitamine A résulté en un accroissement significatif de $38 \%$ du risque de transmission verticale globale du VIH, alors que les multivitamines n'ont pas eu cet effet [74]. Une supplémentation multivitaminique a également significativement réduit le risque de transmission du VIH par l'allaitement maternel et a amélioré la survie sans infection à VIH chez les enfants nés de femmes présentant une altération de l'état nutritionnel ou immunologique (quartile inférieur de la numération totale des lymphocytes) à l'entrée dans l'étude et qui étaient demeurés négatifs pour le VIH à 6 semaines.

Dans l'étude TOV, les investigateurs ont également examiné divers marqueurs cliniques et immunologiques de progression de la maladie pendant toute la durée de la période de suivi post-partum chez des femmes infectées par le VIH. La supplémentation multivitaminique a réduit d'environ $30 \%$ le risque de progression au stade IV de l'OMS et de décès liés au sida ( $R R=0,71$, IC 95\% 0,51, 0,98) [54]. Les multivitamines ont également significativement réduit l'incidence des manifestations orales et digestives d'infections à VIH et des cas rapportés d'as-thénie, d'éruption et d'infections aiguës des voies respiratoires supérieures chez les mères. Les suppléments multivitaminiques ont significativement augmenté les comptes de lymphocytes CD4 et CD8, réduit la charge virale [54] et fait preuve d'un effet protecteur contre l'émaciation chez les femmes infectées par le VIH [75]. Une supplémentation en vitamine A seule n'a pas entrainé de telles améliorations de ces paramètres [73].

Les effets indésirables observés dans le groupe vitamine A de l'essai tanzanien ont pu être imputables à la composante $\beta$-carotène de la supplémentation. Le $\beta$-carotène s'est avéré relativement bien toléré pendant de courtes périodes chez des sujets infectés par le VIH, mais la tolérance d'une supplémentation prolongée n'a pas été étudiée $[76,77]$. Certains aperçus récents sur la tolérance à long terme et l'efficacité thérapeutique des carotènes proviennent d'un essai d'intervention mené chez des adultes atteints d'une forme avancée de sida au Canada, le CTN 091/CRIT Carotenoids Study Group [78]. Les investigateurs de cette étude ont aléatoirement affecté 166 patients au groupe témoin, qui a reçu une supplémentation standard en micronutriments (dont vitamine A et oligoéléments) et 163 patients au groupe traitement, qui a reçu le supplément standard en micronutriments ainsi que des caroténoïdes mixtes (équivalant à $72 \mathrm{mg}$ de $\beta$-carotène) quotidiennement pendant 18 mois. Aucun effet indésirable des caroténoïdes n'a été observé. De fait, une augmentation statistiquement non significative de la mortalité a été observée chez les participants n'ayant pas reçu des caroténoïdes com- 
parativement à ceux ayant reçu ces produits. Cette augmentation est devenue significative après correction en fonction du compte des lymphocytes CD4 et de la concentration sérique en carotène à l'entrée dans l'étude (rapport de risque du temps écoulé jusqu'au décès $=3,15$, IC 95\% 1,10, 8,98). Une limitation majeure de cette étude a cependant été la faible dose de supplément administré. Les auteurs ont indiqué que la dose quotidienne de traitement à l'étude n'avait été équivalente qu'à 9,2 mg de $\beta$-carotène au cours de l'étude au lieu des $72 \mathrm{mg}$ proposés.

L’effet indésirable observé de la vitamine A sur la transmission verticale du VIH lors des essais menés en Tanzanie et au Zimbabwe peut également être dû à l'effet de cette vitamine sur le génome du virus. La vitamine A pourrait potentiellement moduler la réplication du VIH par un élément de réponse à l'acide rétinoïque dans le génome viral [79]. La vitamine A peut également induire une augmentation de la densité des récepteurs CCR5 en accroissant la multiplication et la différenciation des cellules lymphoïdes et myéloïdes. Ces récepteurs CCR 5 sont capitaux pour la fixation du virus aux lymphocytes et la réplication virale consécutive $[71,80]$. De plus, la vitamine A peut accroître le risque de transmission du VIH en accélérant le taux de progression de la maladie chez la mère. Par exemple, lors d'une importante étude observationnelle de cohorte aux États-Unis, les investigateurs ont observé une relation en forme de $U$ entre la consommation alimentaire et supplémentaire de vitamine $A$ et le risque de progression vers un sida clinique et le décès $[81,82]$. Plus spécifiquement, les quartiles inférieurs et supérieurs de la consommation de vitamine A ont été associés à une augmentation significative de la progression vers un sida comparativement aux deux quartiles médians.

Il existe deux distinctions notables entre l'étude TOV menée en Tanzanie et les autres études susmentionnées. Tout d'abord, dans l'essai tanzanien, la supplémentation maternelle s'est poursuivie au-delà de la période anténatale, ce qui accroît la pertinence des observations pour les femmes allaitantes infectées par le VIH et leurs nourrissons. Deuxièmement, les investigateurs ont examiné les effets de suppléments en micronutriments sur des voies spécifiques de transmission verticale, dont in utero, pendant l'accouchement et par l'allaitement maternel. Les observations ont démontré les effets bénéfiques d'une supplémentation en multivitamines sur trois aspects: statut nutritionnel et immunitaire maternel, TME du VIH par l'intermédiaire de l'allaitement et croissance, développement, morbidité et mortalité chez les nourrissons.

\section{Conclusions}

L'allaitement maternel est bénéfique tant pour la mère que pour le nourrisson allaité, avec une amélioration de leur état de santé ainsi que de la croissance et du développement du nourrisson. Cependant, dans le contexte du VIH/sida, l'allai- tement accroît également le risque de TME du VIH de façon proportionnelle à sa durée. Des facteurs supplémentaires exacerbent le risque de transmission verticale du VIH, dont le mode et le type de nutrition du nourrisson, le statut nutritionnel et immunologique de la mère, et le stade de l'infection à VIH chez celle-ci.

Les recommandations de l'Organisation Mondiale de la Santé préconisent l'évitement de l'allaitement par les femmes infectées par le VIH quand une alimentation de remplacement est acceptable, faisable, financièrement abordable, durable et sûre. Dans les pays en développement où ces conditions ne sont pas réunies, l'OMS recommande un allaitement maternel exclusif pendant les 6 premiers mois suivi d'un sevrage immédiat.

Peu d'études ont exploré le rôle de la nutrition chez les femmes allaitantes infectées par le VIH et leurs enfants. Les données actuelles ne sont pas en faveur d'une supplémentation par la vitamine A chez les femmes enceintes ou allaitantes infectées par le VIH, sur la base d'une augmentation du risque de transmission verticale du virus, comme observé lors d'essais menés en Tanzanie et au Zimbabwe. Une supplémentation multivitaminique maternelle quotidienne, incluant vitamines B, C et E, est toutefois fortement recommandée pendant toute la durée des périodes prénatale et post-partum afin de réduire la transmission verticale du VIH, de retarder la progression de l'infection chez la mère et d'améliorer l'état de santé de celle-ci et de son enfant. Cette mesure est probablement particulièrement bénéfique chez les femmes au premier stade de l'infection à VIH et non encore éligibles pour un traitement antirétroviral. L'efficacité et la tolérance de la supplémentation multivitaminique chez les sujets qui reçoivent un traitement antirétroviral doivent être encore confirmées.

\section{Lacunes des recherches et orientations futures}

Des recherches supplémentaires sont justifiées afin d'examiner le rôle de la nutrition sur la santé des femmes allaitantes positives pour le VIH et de leurs enfants. Plus spécifiquement, de nouvelles études sont nécessaires afin de déterminer le mécanisme exact de la transmission du VIH par l'intermédiaire de l'allaitement maternel. Cette identification permettrait de déterminer les facteurs nutritionnels et autres pouvant être les plus pertinents afin de réduire la TME du VIH. L'impact de facteurs nutritionnels sur des situations qui favorisent le risque de transmission du VIH, tels qu'une mastite, doit également être défini.

De nouvelles recherches sont donc nécessaires afin de déterminer la supplémentation adéquate en micronutriments chez les femmes allaitantes infectées par le VIH et leurs nourrissons. En particulier, l'interaction entre le traitement antirétroviral et des facteurs nutritionnels dont une supplémentation en micronutriments doit être élucidée. 
De nouvelles recherches sont également justifiées pour la conception de programmes nutritionnels alternatifs susceptibles d'être efficaces pour les nourrissons. Ce point devient particulièrement important à la lumière de données récentes démontrant la contribution d'une alimentation complémentaire ou de remplacement inadéquate à l'accroissement du risque d'arrêt et de retard de croissance chez les nourrissons nés de femmes infectées par le VIH [83]. Des conseils aux mères allaitantes portant sur leur nutrition et celle de leurs nourrissons sont donc vraiment nécessaires afin d'assurer un arrêt précoce de l'allaitement et l'instauration consécutive de pratiques nutritionnelles adéquates par la suite chez les femmes infectées par le VIH qui ont choisi d'allaiter.

\section{Références}

1 UNAIDS: AIDS Epidemic Update: 2004. Geneva, UNAIDS, 2004.

2 UNAIDS: AIDS Epidemic Update: 2005. Geneva, UNAIDS, 2005

3 Mofenson LM: Successes and challenges in the perinatal HIV-1 epidemic in the United States as illustrated by the HIV-1 serosurvey of childbearing women. Arch Pediatr Adolesc Med 2004;158:422-425.

4 Dabis F, Msellati P, Dunn D, et al: Estimating the rate of mother-to-child transmission of HIV. Report of a workshop on methodological issues Ghent (Belgium), 17-20 February 1992. The Working Group on Mother-toChild Transmission of HIV. AIDS 1993;7: 1139-1148.

5 Coutsoudis A, Dabis F, Fawzi W, et al: Late postnatal transmission of HIV-1 in breastfed children: an individual patient data meta-analysis. J Infect Dis 2004;189:21542166.

6 WHO: New Data on the Prevention of Mother-to-Child Transmission of HIV and Their Policy Implications: Conclusions and Recommendations. Geneva, UNFPA/WHO/ UNAIDS Inter-Agency Task Team on Mother-to-Child Transmission of HIV, 2001

7 Coutsoudis A, Pillay K, Spooner E, et al: Morbidity in children born to women infected with human immunodeficiency virus in South Africa: does mode of feeding matter? Acta Paediatr 2003;92:890-895.

8 Shapiro RL, Lockman S, Thior I, et al: Low adherence to recommended infant feeding strategies among HIV-infected women: results from the pilot phase of a randomized trial to prevent mother-to-child transmission in Botswana. AIDS Educ Prev 2003;15 221-230.

9 Eide M, Myhre M, Lindbaek M, Sundby J, Arimi P, Thior I: Social consequences of HIV-positive women's participation in prevention of mother-to-child transmission programmes. Patient Educ Couns 2006;60: 146-151.

10 Dermer A: Breastfeeding and women's health. J Womens Health 1998;7:427-433.

11 Kuhn L, Stein Z, Susser M: Preventing mother-to-child HIV transmission in the new millennium: the challenge of breast feeding. Paediatr Perinat Epidemiol 2004;18:10-16.
12 Nicoll A, Newell ML, Van Praag E, et al: Infant feeding policy and practice in the presence of HIV-1 infection. AIDS 1995;9:107119.

13 WHO: Effect of breastfeeding on infant and child mortality due to infectious diseases in less developed countries: a pooled analysis. WHO Collaborative Study Team on the Role of Breastfeeding on the Prevention of Infant Mortality. Lancet 2000;355:451-455.

14 Jones G, Steketee RW, Black RE, et al: How many child deaths can we prevent this year? Lancet 2003;362:65-71.

15 Habicht JP, DaVanzo J, Butz WP: Mother's milk and sewage: their interactive effects on infant mortality. Pediatrics 1988;81:456461.

16 Garofalo RP, Goldman AS: Cytokines, chemokines, and colony-stimulating factors in human milk: the 1997 update. Biol Neonate 1998:74:134-142.

17 Goldman AS: The immune system of human milk: antimicrobial, antiinflammatory and immunomodulating properties. Pediatr Infect Dis J 1993;12:664-671.

18 Xanthou M, Bines J, Walker WA: Human milk and intestinal host defense in newborns: an update. Adv Pediatr 1995;42:171208.

19 Gordon N: Nutrition and cognitive function. Brain Dev 1997;19:165-170.

20 Weinberg GA: The dilemma of postnatal mother-to-child transmission of HIV: to breastfeed or not? Birth 2000;27:199-205.

21 Villalpando S, Hamosh M: Early and late effects of breast-feeding: does breast-feeding really matter? Biol Neonate 1998;74:177191.

22 Picciano MF: Pregnancy and lactation: physiological adjustments, nutritional requirements and the role of dietary supplements. J Nutr 2003;133:1997S-2002S.

23 Wilson PR, Pugh LC: Promoting nutrition in breastfeeding women. J Obstet Gynecol Neonatal Nurs 2005;34:120-124.

24 Jensen RG: Handbook of Milk Composition. San Diego, Academic Press, 1995.

25 Ogundele MO, Coulter JB: HIV transmission through breastfeeding: problems and prevention. Ann Trop Paediatr 2003;23:91106.
26 Palasanthiran P, Ziegler JB, Stewart GJ, et al: Breast-feeding during primary maternal human immunodeficiency virus infection and risk of transmission from mother to infant. J Infect Dis 1993;167:441-444.

27 Nduati RW, John GC, Kreiss J: Postnatal transmission of HIV-1 through pooled breast milk. Lancet 1994;344:1432.

28 Temmerman M, Nyongo AO, Bwayo J, et al: Risk factors for mother-to-child transmission of human immunodeficiency virus-1 infection. Am J Obstet Gynecol 1995;172: 700-705.

29 Thorne C, Newell ML: Prevention of mother-to-child transmission of HIV infection. Curr Opin Infect Dis 2004;17:247-252.

30 Mayaux MJ, Blanche S, Rouzioux C, et al: Maternal factors associated with perinatal HIV-1 transmission: the French Cohort Study: 7 years of follow-up observation. The French Pediatric HIV Infection Study Group. J Acquir Immune Defic Syndr Hum Retrovirol 1995;8:188-194.

31 Ometto L, Zanotto C, Maccabruni A, et al: Viral phenotype and host-cell susceptibility to HIV-1 infection as risk factors for motherto-child HIV-1 transmission. AIDS 1995;9: 427-434.

32 Lathey JL, Tsou J, Brinker K, et al: Lack of autologous neutralizing antibody to human immunodeficiency virus type 1 (HIV-1) and macrophage tropism are associated with mother-to-infant transmission. J Infect Dis 1999; 180:344-350.

33 Semba RD, Kumwenda N, Hoover DR, et al: Human immunodeficiency virus load in breast milk, mastitis, and mother-to-child transmission of human immunodeficiency virus type 1. J Infect Dis 1999;180:93-98.

34 John GC, Nduati RW, Mbori-Ngacha DA, et al: Correlates of mother-to-child human immunodeficiency virus type 1 (HIV-1) transmission: association with maternal plasma HIV-1 RNA load, genital HIV-1 DNA shedding, and breast infections. J Infect Dis 2001; 183:206-212.

35 Miller N: HIV and breastfeeding: what we do and do not know in 2005. Midwifery Today Int Midwife 2006 Spring;(77):38-39. 
-36 Miotti PG, Taha TE, Kumwenda NI, et al: HIV transmission through breastfeeding: a study in Malawi. JAMA 1999;282:744-749.

-37 Olayinka B, Oni AO, Mbajiorgu FE: Impact of infant feeding practices on the risk of mother to child transmission of HIV-1 in Zimbabwe. J Paediatr Child Health 2000;36: 313-317.

38 Van de Perre P, Simonon A, Msellati P, et al: Postnatal transmission of human immunodeficiency virus type 1 from mother to infant. A prospective cohort study in Kigali, Rwanda. N Engl J Med 1991;325:593-598.

- 39 Van de Perre P, Hitimana DG, Simonon A, et al: Postnatal transmission of HIV-1 associated with breast abscess. Lancet 1992;339: 1490-1491.

-40 Nduati R, John G, Mbori-Ngacha D, et al: Effect of breastfeeding and formula feeding on transmission of HIV-1: a randomized clinical trial. JAMA 2000;283:1167-1174.

-41 Coutsoudis A, Pillay K, Spooner E, et al: Influence of infant-feeding patterns on early mother-to-child transmission of HIV-1 in Durban, South Africa: a prospective cohort study. South African Vitamin A Study Group. Lancet 1999;354:471-476.

42 Coutsoudis A, Pillay K, Kuhn L, et al: Method of feeding and transmission of HIV-1 from mothers to children by 15 months of age: prospective cohort study from Durban, South Africa. AIDS 2001;15:379-387.

43 Downs JH, Cooper PA: HIV and lactation. Ann Nestlé [Engl] 2007;65:29-38.

44 Papathakis PC, Rollins NC, Brown KH, et al: Comparison of isotope dilution with bioimpedance spectroscopy and anthropometry for assessment of body composition in asymptomatic HIV-infected and HIV-uninfected breastfeeding mothers. Am J Clin Nutr 2005;82:538-546.

45 Nduati R, Richardson BA, John G, et al: Effect of breastfeeding on mortality among HIV-1 infected women: a randomised trial. Lancet 2001;357:1651-1655.

46 Newell M: Does breastfeeding really affect mortality among HIV-1 infected women? Lancet 2001;357:1634-1635.

-47 Coutsoudis A, Coovadia H, Pillay K, Kuhn L: Are HIV-infected women who breastfeed at increased risk of mortality? AIDS 2001;15: 653-655.

-48 Sedgh G, Spiegelman D, Larsen U, et al: Breastfeeding and maternal HIV-1 disease progression and mortality. AIDS 2004;18: 1043-1049.

49 Kuhn L, Kasonde P, Sinkala M, et al: Prolonged breast-feeding and mortality up to two years post-partum among HIV-positive women in Zambia. AIDS 2005;19:16771681.

50 Taha TE, Kumwenda NI, Hoover DR, et al: The impact of breastfeeding on the health of HIV-positive mothers and their children in sub-Saharan Africa. Bull World Health Organ 2006;84:546-554.
51 Breastfeeding and HIV International Transmission Study Group: Mortality among HIV-1-infected women according to children's feeding modality: an individual patient data meta-analysis. J Acquir Immune Defic Syndr 2005;39:430-438.

52 Allard JP, Aghdassi E, Chau J, et al: Effects of vitamin $\mathrm{E}$ and $\mathrm{C}$ supplementation on oxidative stress and viral load in HIV-infected subjects. AIDS 1998;12:1653-1659.

53 Jiamton S, Pepin J, Suttent R, et al: A randomized trial of the impact of multiple micronutrient supplementation on mortality among HIV-infected individuals living in Bangkok. AIDS 2003;17:2461-2469.

54 Fawzi WW, Msamanga GI, Spiegelman D, et al: A randomized trial of multivitamin supplements and HIV disease progression and mortality. N Engl J Med 2004;351:23-32.

- 55 Fawzi W, Msamanga G, Spiegelman D, Hunter DJ: Studies of vitamins and minerals and HIV transmission and disease progression. J Nutr 2005;135:938-944.

56 Dorosko SM: Vitamin A, mastitis, and mother-to-child transmission of HIV-1 through breast-feeding: current information and gaps in knowledge. Nutr Rev 2005;63:332346.

57 Farias EF, Ong DE, Ghyselinck NB, et al: Cellular retinol-binding protein I, a regulator of breast epithelial retinoic acid receptor activity, cell differentiation, and tumorigenicity. J Natl Cancer Inst 2005;97:21-29.

58 Seewaldt VL, Caldwell LE, Johnson BS, et al: Inhibition of retinoic acid receptor function in normal human mammary epithelial cells results in increased cellular proliferation and inhibits the formation of a polarized epithelium in vitro. Exp Cell Res 1997;236:1628.

59 Dreyfuss ML, Fawzi WW: Micronutrients and vertical transmission of HIV-1. Am J Clin Nutr 2002;75:959-970.

60 Buck J, Ritter G, Dannecker L, et al: Retinol is essential for growth of activated human $B$ cells. J Exp Med 1990;171:1613-1624.

61 Garbe A, Buck J, Hammerling U: Retinoids are important cofactors in T cell activation. J Exp Med 1992;176:109-117.

62 Kuhn L, Coutsoudis A, Moodley D, et al: Thelper cell responses to HIV envelope peptides in cord blood: protection against intrapartum and breast-feeding transmission. AIDS 2001;15:1-9.

63 Emmett PM, Rogers IS: Properties of human milk and their relationship with maternal nutrition. Early Hum Dev 1997;49(suppl): S7-S28.

64 Nduati RW, John GC, Richardson BA, et al: Human immunodeficiency virus type 1-infected cells in breast milk: association with immunosuppression and vitamin A deficiency. J Infect Dis 1995;172:1461-1468.
65 Semba RD, Miotti PG, Chiphangwi JD, et al: Maternal vitamin A deficiency and motherto-child transmission of HIV-1. Lancet 1994; 343:1593-1597.

66 Baeten JM, Richardson BA, Bankson DD, et al: Use of serum retinol-binding protein for prediction of vitamin A deficiency: effects of HIV-1 infection, protein malnutrition, and the acute phase response. Am J Clin Nutr 2004;79:218-225.

67 Filteau SM, Morris SS, Abbott RA, et al: Influence of morbidity on serum retinol of children in a community-based study in northern Ghana. Am J Clin Nutr 1993;58: 192-197.

68 Coutsoudis A, Pillay K, Spooner E, et al: Randomized trial testing the effect of vitamin A supplementation on pregnancy outcomes and early mother-to-child HIV-1 transmission in Durban, South Africa. South African Vitamin A Study Group. AIDS 1999; 13:1517-1524.

-69 Pillay K, Coutsoudis A, York D, et al: Cellfree virus in breast milk of HIV-1-seropositive women. J Acquir Immune Defic Syndr 2000;24:330-336.

70 Kumwenda N, Miotti PG, Taha TE, et al: Antenatal vitamin A supplementation increases birth weight and decreases anemia among infants born to human immunodeficiency virus-infected women in Malawi. Clin Infect Dis 2002;35:618-624.

71 Iliff PJ, Piwoz EG, Tavengwa NV, et al: Early exclusive breastfeeding reduces the risk of postnatal HIV-1 transmission and increases HIV-free survival. AIDS 2005;19:699-708.

72 Fawzi WW, Msamanga G, Hunter D, et al: Randomized trial of vitamin supplements in relation to vertical transmission of HIV-1 in Tanzania. J Acquir Immune Defic Syndr 2000;23:246-254.

73 Fawzi WW, Msamanga GI, Spiegelman D, et al: Randomised trial of effects of vitamin supplements on pregnancy outcomes and $\mathrm{T}$ cell counts in HIV-1-infected women in Tanzania. Lancet 1998;351:1477-1482.

74 Fawzi WW, Msamanga GI, Hunter D, et al: Randomized trial of vitamin supplements in relation to transmission of HIV-1 through breastfeeding and early child mortality. AIDS 2002;16:1935-1944.

75 Villamor E, Saathoff E, Manji K, et al: Vitamin supplements, socioeconomic status, and morbidity events as predictors of wasting in HIV-infected women from Tanzania. Am J Clin Nutr 2005;82:857-865.

76 Coodley GO, Nelson HD, Loveless MO, Folk C: Beta-carotene in HIV infection. J Acquir Immune Defic Syndr 1993;6:272-276.

77 Nimmagadda AP, Burri BJ, Neidlinger T, et al: Effect of oral beta-carotene supplementation on plasma human immunodeficiency virus (HIV) RNA levels and CD4+ cell counts in HIV-infected patients. Clin Infect Dis 1998;27:1311-1313. 
78 Austin J, Singhal N, Voigt R, et al: A community randomized controlled clinical trial of mixed carotenoids and micronutrient supplementation of patients with acquired immunodeficiency syndrome. Eur J Clin Nutr 2006;60:1266-1276.

79 Semba RD, Lyles CM, Margolick JB, et al: Vitamin A supplementation and human immunodeficiency virus load in injection drug users. J Infect Dis 1998;177:611-616.
80 MacDonald KS, Malonza I, Chen DK, et al: Vitamin A and risk of HIV-1 seroconversion among Kenyan men with genital ulcers. AIDS 2001;15:635-639.

81 Tang AM, Graham NM, Kirby AJ, et al: Dietary micronutrient intake and risk of progression to acquired immunodeficiency syndrome (AIDS) in human immunodeficiency virus type 1 (HIV-1)-infected homosexual men. Am J Epidemiol 1993;138:937951.
82 Tang AM, Graham NM, Saah AJ: Effects of micronutrient intake on survival in human immunodeficiency virus type 1 infection. Am J Epidemiol 1996;143:1244-1256.

83 Becquet R, Leroy V, Ekouevi DK, et al: Complementary feeding adequacy in relation to nutritional status among early weaned breastfed children who are born to HIV-infected mothers: ANRS 1201/1202 Ditrame Plus, Abidjan, Cote d'Ivoire. Pediatrics 2006; 117:e701-e710. 